\title{
Cystic Lymphangioma of Pancreas - Case Series
}

\author{
Dr.Arun.K ${ }^{1}$, R.Ravi ${ }^{2}$, Senthilkumar perumal ${ }^{2}$, R.Kamalakannan ${ }^{2}$, \\ Umamaheshwaran $^{2}$, J.Saravanan ${ }^{2}$, M.Thiruvarul ${ }^{2}$, C.Sugumar ${ }^{3}$, \\ S.Jeswanth ${ }^{3}$, P.Ravichandran ${ }^{3}$, \\ ${ }^{I} P G$ Residentin Surgical Gastroenterology., \\ ${ }^{2}$ Assistant professors in surgical gastroenterology \\ ${ }^{3}$ Professors in surgical gastroenterology \\ Institute of Surgical Gastroenterology\&Liver Transplantation. \\ Government Stanley Medical College \& Hospital. Chennai Tamilnadu India.
}

\section{Highlights}

Cystic lymphangiomaof Pancreas is a rare entity.

$>$ Eventhough imaging shows large size, mass wont be palpable because of yielding nature.

$>$ Importance of this entity lies in the differential diagnosis from other cystic lesions of pancreas based on age at presentation, clinical \& radiological characteristics.

\section{Introduction}

Cysticlymphangioma of pancreas is a very rare entity ${ }^{1}$. In 1913, Koch reportedthe first case of cystic lymphangioma of pancreas ${ }^{1}$.Lymphangioma usually occurs in the neck, axillary region, and rarely in the mediastinum. Abdominal organs are uncommon sites of origin for lymphangioma ${ }^{1}$. Of all the lymphangiomas in the peritoneal cavity, about $70 \%$ have been found in the mesentery of small intestine ${ }^{2}$. Pancreas is one of the rarest site\& it is to be considered as one of the differential diagnosis among cystic neoplasms of pancreas, pseudocyst of pancreas \& also other retroperitoneal cystic tumours.

\section{Case Series - Study}

Herein, We report 6 cases of cystic pancreatic lymphangioma from march 2013 to june 2016 . All patients presented in $2^{\text {nd }}$ decade as vague epigastric discomfort.Male : female ratio is 1:1; more commonly located in the region of body \& tail of the pancreas (body \& tail - 4 ; head - 2 ) occupying lesser sac $\&$ upper retroperitoneum. Upon physicalexamination, there was no palpable mass because of yielding nature though lesion was large.Size of the lesion vary from $6 \mathrm{~cm}$ to $19 \mathrm{~cm}$ (average- $12 \mathrm{~cm}$ ) with Hepatic artery \&GDA seen coursing through the lesion in one case.

Radiological Imaging studies commonly revealed multiseptated cystic mass lesion located in the upper ventral pancreatic area (head - 2; body\&tail -4)neighboring posterior stomach occupying upper retroperitoneum; Size of the lesion vary from $6 \mathrm{~cm}$ to $19 \mathrm{~cm}$ (average- $12 \mathrm{~cm}$ ) with Hepatic artery \& GDA seen coursing through the lesion in one case.EUS guided cyst fluid aspiration done \& level of CEA,Amylase,

CA 19-9 are found to be within normal limits.Complete excision preserving vessels was done in all the cases.Final pathological diagnosis was reported as cystic lymphangioma.All the patients made complete recovery \& no recurrences on followup.

$\underline{\text { Table }}$

\begin{tabular}{|l|l|l|l|l|l|l|}
\hline & Case-1 & Case-2 & Case-3 & Case-4 & Case-5 & Case-6 \\
\hline Age & 16 & 12 & 20 & 13 & 19 & 16 \\
\hline Sex & Female & Male & Male & Female & Male & Female \\
\hline Location & Head & Head & Body \& tail & Body \&tail & Body \& tail & Body \& tail \\
\hline Extent & $\begin{array}{l}\text { Lesser sac } \\
\text { \&HDL } \\
\text { ligament }\end{array}$ & $\begin{array}{l}\text { Lesser } \\
\text { sac }\end{array}$ & $\begin{array}{l}\text { Lesser sac } \\
\text { Upper } \\
\text { retroperitoneum }\end{array}$ & $\begin{array}{l}\text { Lesser sac } \\
\text { Upper } \\
\text { retroperitoneum }\end{array}$ & $\begin{array}{l}\text { Lesser sac } \\
\text { Upper } \\
\text { retroperitoneum }\end{array}$ & $\begin{array}{l}\text { Lesser sac } \\
\text { Upper } \\
\text { retroperitoneum }\end{array}$ \\
\hline Complication & Infection & - & - & - & - & - \\
\hline
\end{tabular}


Figure -1

CECT abdomen showing multiseptatedhypodense cystic lesion with nonenhancing thin delicate septa occupying the body \& tail of the pancreas in the lesser sac.

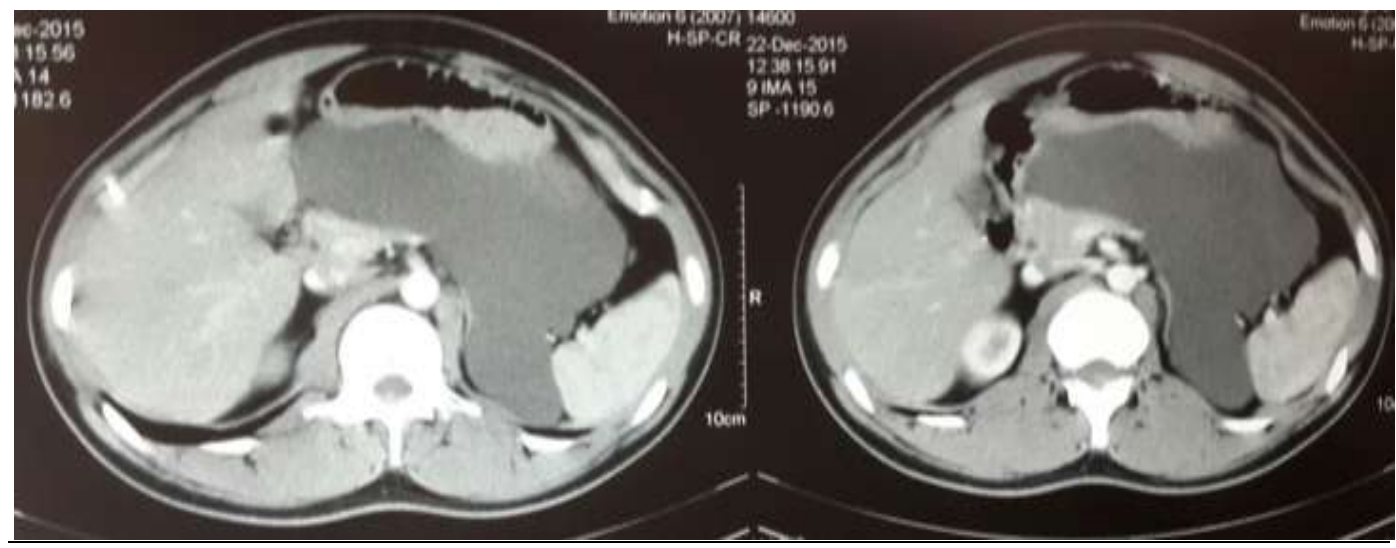

Figure-2

Intraoperative picture showing cystic lymphangioma occupying the body \& tail of the pancreas in the lesser sac posterior to stomach.

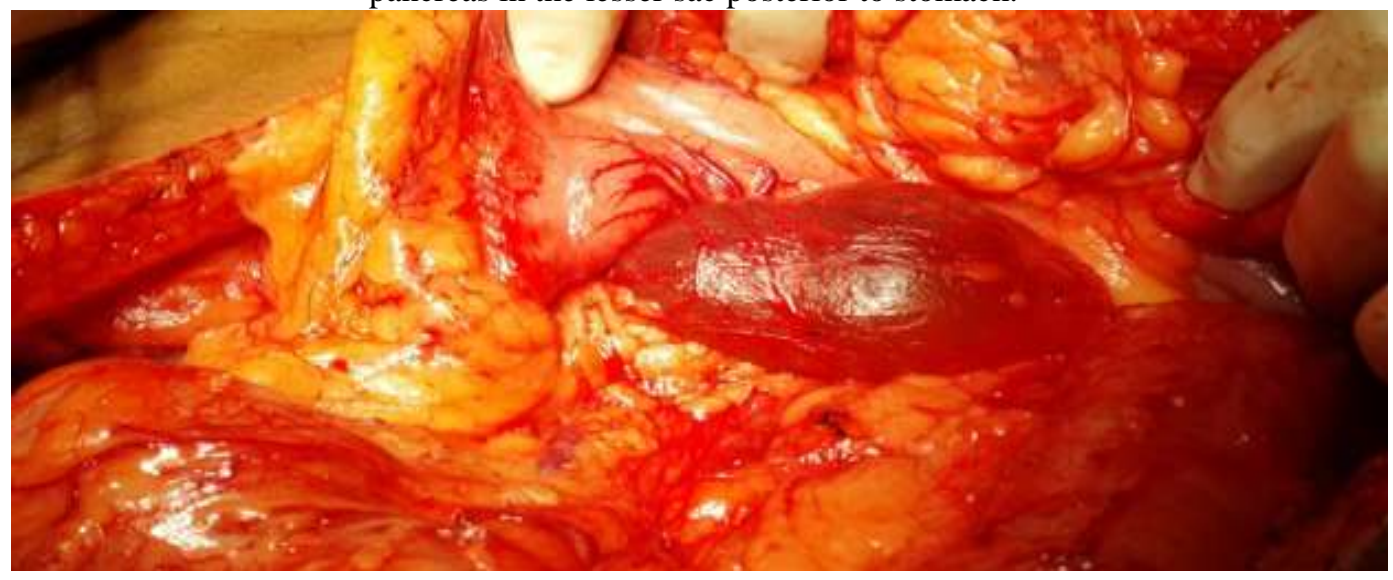

III. Discussion

Cystic lymphangiomas are multi-loculated soft cystic masses, composed of dilated lymphatic channels, divided by thin septae because of insufficient drainage due to abdominal trauma, inflammatory process, surgery or radiation therapy ${ }^{3}$.Pancreatic lymphangioma is a very rare entity less than $1 \%$.In our study lesion more commonly located in body \& tail of the pancreas (body \& tail - 4; head -2); male : female ratio-1:1; Age group $-2^{\text {nd }}$ decade; Size of the lesion varying from $6 \mathrm{~cm}$ to $19 \mathrm{~cm}$ (average- $12 \mathrm{~cm}$ ) ; occupying upper retroperitoneum; not invading any vascular structures though imaging suggested hepatic artery \& GDA coursing through the lesion in one case. Infection as a complication was found in one case althoughintracystic hemorrhage, cyst rupture, volvulus reported in literature ${ }^{4}$. Association of these lesions with blue rubber bleb nevus syndrome has also been described in literature. ${ }^{5}$

Importance of this entity is that this has to be differentiated from Pseudocysts, cystic neoplasm of pancreas (mucinous and serouscystadenomas, solid pseudopapillarytumors,IPMN),congenital cysts and ductal carcinoma\& other retroperitoneal cystic tumours ${ }^{6}$.Imaging studies (CT ) helped us to differentiate these lesions from others. These lesions usuallypresented asmultiloculated cystic tissue lesion with anechoic contents with nonenhancing thin delicate septa crisscrossing the lesion ${ }^{7}$; No true capsule; No mural nodules/ wall calcification or perilesional lymphadenopathy .Complete excision is the treatment of choice ; recurrence can occur in $7 \%$ of the cases ${ }^{8}$ though no recurrences on follow up. Although lymphangioma rarely becomes malignant, its prognosis is generally good ${ }^{9}$

\section{Conclusion}

Cystic pancreatic lymphangiomas are rarely seen tumors. Importance of this entity lies in the differential diagnosis from other cystic lesions of pancreas based on age at presentation,clinical \& radiological characteristics.Complete surgical resection is curative. 


\section{References}

[1]. Kawaguchi K, Motoi F, Ohtsuka H, EtAl.CysticLymphangioma Of The Pancreas With Spontaneous Rupture: Report Of A Case. Case Rep Gastroenterol2011;5:288-94

[2]. Kim JH, Ryu WS, Min BW, Et Al. Acquired Omental Cystic LymphangiomaAfterSubtotalgastrectomy: A Case Report. J KoreanmedSci2009;24:1212-5.

[3]. Fahimi H, Faridi M, Gholamin S, Et Al. Cystic Lymphangioma Of The Pancreas:

[4]. Diagnostic And Therapeutic Challenges.JOP2010;11:617-9. Erratum In: JOP 2010;11:619.

[5]. Bhavsar T, Saeed-Vafa D, HarbisonS,Inniss S. Retroperitoneal Cystic Lymphangioma In An Adult: A Case Report And Review Of The Literature. World J GastrointestPathophysiol2010

[6]. Nobuhara Y, Onoda N, Fukai K, Hosomi N, Ishii M, WakasaK,Et Al. TIE2 Gain-Of-Function Mutation In A Patient With Pancreatic Lymphangioma Associated With Blue Rubber-Bleb Nevus Syndrome:Report Of A Case. Surg Today 2006

[7]. Khandelwal M, Lichtenstein GR, Morris JB, Furth EE, Long WB. Abdominal Lymphangioma Masquerading As A Pancreatic Cystic Neoplasm. J Clin Gastroenterol 1995

[8]. Colovic RB, Grubor NM, Micev MT, Et Al.CysticLymphangioma Of The Pancreas. World J Gastroenterol2008;14:6873-5.

[9]. Surlin V, Georgescu E, Dumitrescu C, Et Al. RetropancreaticCystic Lymphangioma -Considerations Upon A Case. Rom JMorpholEmbryol2011;52Suppl1:493-6.

[10]. Chung SH, Park YS, Jo YJ, Et Al. Asymptomatic Lymphangioma Involving The Spleen And Retroperitoneum In Adults. World J Gastroenterol2009; 15:5620-3

\begin{tabular}{|l|l|l|c|}
\hline & Speciality & Institution & Email address \\
\hline 1.Prof.P.Ravichandran & M.B.B.S.,M.S.,M.ch & $\begin{array}{l}\text { Institute of Surgical Gastroenterology\& Liver } \\
\text { Transplantation. } \\
\text { Govt Stanley Medical college \& Hospital }\end{array}$ & prahari05@gmail.com \\
\hline 2.Prof.S.Jeswanth & M.B.B.S.,M.S.,M.ch & $\begin{array}{l}\text { Institute of Surgical Gastroenterology\& Liver dr_jeswanth@yahoo.co.in } \\
\text { Transplantation. } \\
\text { Govt Stanley Medical college \& Hospital }\end{array}$ & $\begin{array}{l}\text { Institute of Surgical Gastroenterology\& Liver dr.sugumar@yahoo.com } \\
\text { Transplantation. } \\
\text { Govt Stanley Medical college \& Hospital }\end{array}$ \\
\hline 3.Prof.C.Sugumar & M.B.B.S.,M.S.,M.ch & \\
\hline
\end{tabular}

\begin{tabular}{|l|l|l|l|}
\hline Authors & Speciality & Institution & Email address \\
\hline 1.Arun kasi & $\begin{array}{l}\text { M.B.B.S.,M.S.,M.ch } \\
\text { ( PG resident) }\end{array}$ & $\begin{array}{l}\text { Institute of } \\
\text { Surgical Gastroenterology\& Liver } \\
\text { Transplantation. } \\
\text { Govt Stanley Medical college \& Hospital }\end{array}$ & drkarunms@ gmail.com \\
\hline 2.R.Ravi & M.B.B.S.,M.S.,M.ch & $\begin{array}{l}\text { Institute of Surgical Gastroenterology \& } \\
\text { Liver Transplantation. } \\
\text { Govt Stanley Medical college \& Hospital }\end{array}$ & ravigisurgery@yahoo.com \\
\hline 3.Senthil kumarperumal & M.B.B.S.,M.S.,M.ch & $\begin{array}{l}\text { Institute of Surgical Gastroenterology \& } \\
\text { Liver Transplantation. } \\
\text { Govt Stanley Medical college \& Hospital }\end{array}$ & dr.psenthil@yahoo.com \\
\hline 4.R.Kamalakannan & M.B.B.S.,M.S.,M.ch & $\begin{array}{l}\text { Institute of Surgical Gastroenterology \& } \\
\text { Liver Transplantation. } \\
\text { Govt Stanley Medical college \& Hospital }\end{array}$ & kkgisur@gmail.com \\
\hline 5.J.Saravanan & M.B.B.S.,M.S.,M.ch & $\begin{array}{l}\text { Institute of Surgical Gastroenterology\& } \\
\text { Liver Transplantation. } \\
\text { Govt Stanley Medical college \& Hospital }\end{array}$ & saran_world@yahoo.com \\
\hline 6.Umamaheshwaran & M.B.B.S.,M.S.,M.ch & $\begin{array}{l}\text { Institute of Surgical Gastroenterology\& } \\
\text { Liver Transplantation. } \\
\text { Govt Stanley Medical college \& Hospital }\end{array}$ & dr_ums@yahoo.co.in \\
\hline 7.M.Thiruvarul & M.B.B.S.,M.S.,M.ch & $\begin{array}{l}\text { Institute of Surgical Gastroenterology\& } \\
\text { Liver Transplantation. } \\
\text { Govt Stanley Medical college \& Hospital }\end{array}$ & drmtarul@gmail.com \\
\hline 8.Prof.S.Jeswanth & M.B.B.S.,M.S.,M.ch & $\begin{array}{l}\text { Institute of Surgical Gastroenterology\& } \\
\text { Liver Transplantation. } \\
\text { Govt Stanley Medical college \& Hospital }\end{array}$ & dr_jeswanth@yahoo.co.in \\
\hline 9.Prof.C.Sugumar & M.B.B.S.,M.S.,M.ch & $\begin{array}{l}\text { Institute of Surgical Gastroenterology\& } \\
\text { Liver Transplantation. } \\
\text { Govt Stanley Medical college \& Hospital }\end{array}$ & $\begin{array}{l}\text { dr.sugumar@yahoo.com } \\
\text { Liver Transplantation. } \\
\text { Govt Stanley Medical college \& Hospital }\end{array}$ \\
\hline 10.Prof.P.Ravichandran & M.B.B.S.,M.S.,M.ch & $\begin{array}{l}\text { Instuten of Surgical Gastroenterology\& } \\
\text { prahari05@gmail.com }\end{array}$ \\
\hline
\end{tabular}

